\title{
Up sector of minimal flavor violation: top quark properties and direct $D$ meson $C P$ violation
}

\author{
Yang Bai, ${ }^{a}$ Joshua Berger, ${ }^{b}$ JoAnne L. Hewett ${ }^{b}$ and Ye $\mathbf{L i}^{b}$ \\ ${ }^{a}$ Department of Physics, University of Wisconsin, \\ Madison, WI 53706, U.S.A. \\ ${ }^{b}$ SLAC National Accelerator Laboratory, \\ 2575 Sand Hill Road, Menlo Park, CA 94025, U.S.A. \\ E-mail: yangbai@physics.wisc.edu, jberger@slac.stanford.edu, \\ hewett@slac.stanford.edu, yli@slac.stanford.edu
}

ABstract: Minimal Flavor Violation in the up-type quark sector leads to particularly interesting phenomenology due to the interplay of flavor physics in the charm sector and collider physics from flavor changing processes in the top sector. We study the most general operators that can affect top quark properties and $D$ meson decays in this scenario, concentrating on two $C P$ violating operators for detailed studies. The consequences of these effective operators on charm and top flavor changing processes are generically small, but can be enhanced if there exists a light flavor mediator that is a Standard Model gauge singlet scalar and transforms under the flavor symmetry group. This flavor mediator can satisfy the current experimental bounds with a mass as low as tens of $\mathrm{GeV}$ and explain observed $D$-meson direct $C P$ violation. Additionally, the model predicts a non-trivial branching fraction for a top quark decay that would mimic a dijet resonance.

KeYwords: Beyond Standard Model, CP violation

ARXIV EPRINT: 1305.5537 


\section{Contents}

1 Introduction 1

2 Up sector operators and models 3

2.1 Phenomenology of the effective operators 5

$\begin{array}{lll}2.2 & \text { Light mediators } & 7\end{array}$

3 Top quark properties $\quad 8$

3.1 Single top production 8

$3.2 t \bar{t}$ pair-production $\quad 9$

$\begin{array}{lll}3.3 & \text { Non-standard top decays } & 10\end{array}$

4 Additional constraints and D-meson physics 11

4.1 Enhanced $b$ production 11

$\begin{array}{lll}4.2 & D \text { meson direct } C P \text { violation } & 13\end{array}$

5 Discussion and conclusions $\quad 16$

A Calculation of the partial width $\Gamma\left(Z \rightarrow q \bar{q}^{\prime} \phi\right) \quad 17$

$\begin{array}{lr}\text { B Wilson coefficient running } & 18\end{array}$

$\begin{array}{lr}\text { C Hadronic matrix element estimation } & 18\end{array}$

\section{Introduction}

Low scale extensions of the Standard Model (SM) are forced to contend with the so-called New Physics Flavor Puzzle (NPFP): new physics at or below the TeV scale must have nongeneric flavor structure to satisfy experimental constraints. This problem is exacerbated by the recent discovery of a SM-like Higgs boson [1, 2], which lowers the scale of new physics required to have a natural solution to the hierarchy problem. In order to solve the NPFP, a model of new physics must either be unnatural, with a high scale, or have some mechanism that strongly suppresses low-energy flavor violating interactions, such as flavor blindness, alignment or Minimal Flavor Violation (MFV) [3, 4] . Within MFV, the flavor constraints on the scale of new physics can be reduced from $\mathcal{O}\left(10^{3} \mathrm{TeV}\right)$ to $\mathcal{O}$ (few $\mathrm{TeV}$ ). While there are many studies of the down-type quark sector in the MFV framework [416], less attention has been paid to the up-type sector. In this paper, we explore several interesting phenomena of MFV models in the up-type quark sector.

By treating the SM Yukawa couplings as spurions, MFV provides a systematic way to classify the effective higher-dimensional flavor-violating operators. One can then determine the most important operators for a given process based on the dimension of the 
operators and the Yukawa matrix insertions. One interesting feature of MFV is that the same operator can relate flavor-changing process predictions for one generation to those for another generation, as has already been observed in the down-type quark sector with the correlation of $K^{0}-\bar{K}^{0}$ and $B^{0}-\bar{B}^{0}$ mixings. Applying MFV to the up-type quark sector, the correlations become more interesting because of the large mass gap between the charm quark and top quark masses. Low-energy tests of charm quark flavor violation can be directly related to top quark properties probed in high-energy experiments, including the Large Hadron Collider (LHC). The main focus of this paper is to explore this correlation on phenomena at different energy scales.

Both $\Delta F=2$ and $\Delta F=1$ processes are predicted from the MFV operator analysis. In the up-quark sector, generic $\Delta F=2$ operators are severely constrained by $D^{0}-\bar{D}^{0}$ mixing [17]. In MFV, the relevant operators for $D^{0}-\bar{D}^{0}$ mixing are suppressed by both the bottom quark Yukawa coupling and Cabibbo-Kobayashi-Maskawa (CKM) mixing angles; they are much more weakly constrained. Modifications of top quark properties are therefore suppressed by the cutoff of the effective operator as well as by CKM mixing angles, although an interesting signature of same-sign top pairs could be generated at colliders. Bounds from $D^{0}-\bar{D}^{0}$ mixing are sufficient to constrain $\Delta T=2$ operators such that no accessible collider phenomenology is allowed.

This work therefore focuses on $\Delta F=1 \mathrm{MFV}$ operators in the up-type quark sector. One immediate consequence of such operators is that the decays of both $D$ mesons and top quarks can be modified. In the SM, the decays of these particles are unsuppressed by CKM angles. If the new physics operators are generated by integrating out a heavy particle above a few hundred $\mathrm{GeV}$, the new contributions to these decays are negligibly small, even if one considers sensitive $C P$ violating observables. The story is different if there is a new electroweak and color singlet particle $\phi$ lighter than the top quark. Effective operators can still describe the new physics contribution to $D$-meson decays, but the cutoff scales of the relevant operators can now be as low as $\mathcal{O}(10 \mathrm{GeV})$. The top quark, on the other hand, can directly decay to $\phi$ and a light quark (a similar decay into a charged Higgs plus $b$-quark has been studied in ref. [18]). This new and potentially large decay channel for the top quark is currently allowed and requires a dedicated search at the LHC. The branching ratio and search strategy could be dramatically different from the effective operator analysis in ref. [19].

Light neutral scalars commonly arise as pseudo-Nambu-Goldstone bosons from spontaneous breaking of a global symmetry and as scalars, which may be elementary or composite, in some hidden sector (see ref. [20] for the effects on $B$ physics in this scenario). If the global $\mathrm{SU}(3)^{5}$ flavor symmetry in the $\mathrm{SM}$ is spontaneously broken and a small explicit breaking is added, "light familons" [21] are generic predictions, particularly within the MFV framework. The existence of a light $\phi$ charged under the flavor symmetry is well motivated from this perspective. In this paper, rather than explore the symmetry breaking mechanism of the global flavor symmetry, we study the phenomenological consequences of the light $\phi$ field for $\Delta C=1$ and $\Delta T=1$ processes.

This paper is organized as follows. In section 2, we first classify all four-fermion $\Delta F=1$ operators in MFV involving up-type quarks and introduce the light $\phi$ field that 
can generate large coefficients for these operators. We then study modifications of top quark properties in section 3, including single top production, $t \bar{t}$ pair-production, and nonstandard decays of the top quark. In section 4 , we calculate predictions for several $b$-quark and $c$-quark related observables. Particular attention is devoted to direct $C P$ violation of the neutral $D$ meson. The discussion of UV completions of this model and the conclusions of this work are presented in section 5. A calculation of the partial width $\Gamma\left(Z \rightarrow q \bar{q}^{\prime} \phi\right)$ is discussed in appendix A, running of the relevant Wilson coefficients in appendix B, and hadronic matrix element estimation in appendix C.

\section{Up sector operators and models}

In practice, the principle of MFV is implemented by treating the SM Yukawa matrices as spurions of flavor symmetry. A MFV operator can then be written down by demanding that it is formally flavor invariant. The quark sector before introducing the Yukawa couplings has a global flavor symmetry

$$
G_{F}^{\mathrm{EW}}=\mathrm{SU}(3)_{Q_{L}} \times \mathrm{SU}(3)_{u_{R}} \times \mathrm{SU}(3)_{d_{R}} \times \mathrm{U}(1)_{B} \times \mathrm{U}(1)_{Y} \times \mathrm{U}(1)_{P Q},
$$

where $\mathrm{U}(1)_{B}$ is global baryon symmetry, $\mathrm{U}(1)_{Y}$ is gauge hypercharge symmetry, and $\mathrm{U}(1)_{P Q}$ is Peccei-Quinn symmetry. In the $\mathrm{SM}$, the $\mathrm{U}(1)_{P Q}$ is explicitly broken by the Yukawa couplings, while in MSSM-like two Higgs doublet models, the Yukawa couplings preserve the $\mathrm{U}(1)_{P Q}$ symmetry by assigning opposite charges for $H_{d}$ and down-type quarks. Concentrating on the non-Abelian global symmetries, the SM Yukawa matrices can be treated as spurions with representations

$$
Y_{U} \sim(3, \overline{3}, 1), \quad Y_{D} \sim(3,1, \overline{3}) .
$$

where " $U$ " represents $(u, c, t)$ quarks and " $D$ " represents $(d, s, b)$ quarks.

The description above is the standard description of MFV. There is, however, an equivalent formulation that will be more convenient for studying particles with mass $m \ll v$, where $v$ denotes the Higgs Vacuum Expectation Value (VEV). In this second approach, which we refer to as the EW approach, we construct operators invariant only under the $\mathrm{U}(1)_{E M}$ subgroup of the electroweak gauge group $\mathrm{SU}(2)_{L} \times \mathrm{U}(1)_{Y}$. Any UV completion will, of course, generate $\mathrm{SU}(2)_{L} \times \mathrm{U}(1)_{Y}$ invariant operators, but in the limit we are considering, we can postpone such high energy considerations. One consequence of the EW approach is that the left-handed quark fields can be rotated separately. Couplings to the $W$ boson provide additional flavor violation. The flavor structure can be described by the group

$$
G_{F}^{\mathrm{EW}}=\mathrm{SU}(3)_{u_{L}} \times \mathrm{SU}(3)_{d_{L}} \times \mathrm{SU}(3)_{u_{R}} \times \mathrm{SU}(3)_{d_{R}}
$$

under which there are spurions

$$
\lambda_{U} \sim(3,1, \overline{3}, 1), \quad \lambda_{D} \sim(1,3,1, \overline{3}), \quad V \sim(3, \overline{3}, 1,1) .
$$

We are free to choose a basis where $\lambda_{U}=\operatorname{diag}\left\{\lambda_{\mathrm{u}}, \lambda_{\mathrm{c}}, \lambda_{\mathrm{t}}\right\}, \lambda_{D}=\operatorname{diag}\left\{\lambda_{\mathrm{d}}, \lambda_{\mathrm{s}}, \lambda_{\mathrm{b}}\right\}$, and $V$ is the CKM matrix. Up to $\mathcal{O}\left(\lambda_{U}^{2}, \lambda_{D}^{2}, \lambda_{D} \lambda_{U}\right)$, we present all MFV $\Delta F=1$ operators in 


\begin{tabular}{|l|}
\hline Operator \\
\hline$\left(\bar{u}_{L \alpha} V \gamma^{\mu} d_{L \alpha}\right)\left(\bar{d}_{L \beta} V^{\dagger} \gamma_{\mu} u_{L \beta}\right)$ \\
$\left(\bar{u}_{L \alpha} V \gamma^{\mu} d_{L \beta}\right)\left(\bar{d}_{L \beta} V^{\dagger} \gamma_{\mu} u_{L \alpha}\right)$ \\
$\left(\bar{u}_{R \alpha} \lambda_{U}^{\dagger} V d_{L \alpha}\right)\left(\bar{d}_{L \beta} V^{\dagger} \lambda_{U} u_{R \beta}\right)$ \\
$\left(\bar{u}_{R \alpha} \lambda_{U}^{\dagger} V d_{L \beta}\right)\left(\bar{d}_{L \beta} V^{\dagger} \lambda_{U} u_{R \alpha}\right)$ \\
$\left(\bar{u}_{L \alpha} V \lambda_{D} d_{R \alpha}\right)\left(\bar{d}_{R \beta} \lambda_{D}^{\dagger} V^{\dagger} u_{L \beta}\right)$ \\
$\left(\bar{u}_{L \alpha} V \lambda_{D} d_{R \beta}\right)\left(\bar{d}_{R \beta} \lambda_{D}^{\dagger} V^{\dagger} u_{L \alpha}\right)$ \\
$\left(\bar{u}_{L \alpha} V \lambda_{D} \lambda_{D}^{\dagger} V^{\dagger} \gamma^{\mu} u_{L \alpha}\right)\left(\bar{u}_{R \beta} \gamma_{\mu} u_{R \beta}\right)$ \\
$\left(\bar{u}_{L \alpha} V \lambda_{D} \lambda_{D}^{\dagger} V^{\dagger} \gamma^{\mu} u_{L \beta}\right)\left(\bar{u}_{R \beta} \gamma_{\mu} u_{R \alpha}\right)$ \\
$\left(\bar{u}_{L \alpha} V \lambda_{D} \lambda_{D}^{\dagger} V^{\dagger} \gamma^{\mu} u_{L \alpha}\right)\left(\bar{d}_{R \beta} \gamma_{\mu} d_{R \beta}\right)$ \\
$\left(\bar{u}_{L \alpha} V \lambda_{D} \lambda_{D}^{\dagger} V^{\dagger} \gamma^{\mu} u_{L \beta}\right)\left(\bar{d}_{R \beta} \gamma_{\mu} d_{R \alpha}\right)$ \\
$\left(\bar{u}_{L \alpha} V \gamma^{\mu} d_{L \alpha}\right)\left(\bar{e}_{L} U^{\dagger} \gamma_{\mu} \nu_{L}\right)$ \\
$\left(\bar{u}_{L \alpha} V \lambda_{D} \lambda_{D}^{\dagger} V^{\dagger} \gamma^{\mu} u_{L \alpha}\right)\left(\bar{e}_{L} \gamma_{\mu} e_{L}\right)$ \\
$\left(\bar{u}_{L \alpha} V \lambda_{D} \lambda_{D}^{\dagger} V^{\dagger} \gamma^{\mu} u_{L \alpha}\right)\left(\bar{\nu}_{L} \gamma_{\mu} \nu_{L}\right)$ \\
$\left(\bar{u}_{L \alpha} V \lambda_{D} \lambda_{D}^{\dagger} V^{\dagger} \gamma^{\mu} u_{L \alpha}\right)\left(\bar{e}_{R} \gamma_{\mu} e_{R}\right)$ \\
\hline
\end{tabular}

\begin{tabular}{|ll|}
\hline Operator & Name \\
\hline$\left(\bar{u}_{L \alpha} \gamma^{\mu} V d_{L \alpha}\right)\left(\bar{d}_{R \beta} \gamma_{\mu} \lambda_{D}^{\dagger} V^{\dagger} \lambda_{U} u_{R \beta}\right)$ & $\mathcal{O}_{V 1}$ \\
$\left(\bar{u}_{L \alpha} \gamma^{\mu} V d_{L \beta}\right)\left(\bar{d}_{R \beta} \gamma_{\mu} \lambda_{D}^{\dagger} V^{\dagger} \lambda_{U} u_{R \alpha}\right)$ & $\mathcal{O}_{V 2}$ \\
$\left(\bar{u}_{R \alpha} \lambda_{U}^{\dagger} V d_{L \alpha}\right)\left(\bar{d}_{R \beta} \lambda_{D}^{\dagger} V^{\dagger} u_{L \beta}\right)$ & $\mathcal{O}_{S 1}$ \\
$\left(\bar{u}_{R \alpha} \lambda_{U}^{\dagger} V d_{L \beta}\right)\left(\bar{d}_{R \beta} \lambda_{D}^{\dagger} V^{\dagger} u_{L \alpha}\right)$ & $\mathcal{O}_{S 2}$ \\
$\left(\bar{u}_{L \alpha} V \lambda_{D} d_{R \alpha}\right)\left(\bar{e}_{R} \lambda_{E}^{\dagger} U^{\dagger} \nu_{L}\right)$ & \\
$\left(\bar{u}_{R \alpha} \lambda_{U}^{\dagger} V d_{L \alpha}\right)\left(\bar{e}_{R} \lambda_{E}^{\dagger} U^{\dagger} \nu_{L}\right)$ & \\
$\left(\bar{u}_{L \alpha} V \lambda_{D} d_{R \alpha}\right)\left(\bar{e}_{L} U^{\dagger} \lambda_{\nu}^{\dagger} \nu_{R}\right)$ & \\
$\left(\bar{u}_{R \alpha} \lambda_{U}^{\dagger} V d_{L \alpha}\right)\left(\bar{e}_{L} U^{\dagger} \lambda_{\nu}^{\dagger} \nu_{R}\right)$ & \\
\hline
\end{tabular}

Table 1. A complete list of four-fermion operators mediating $\Delta F=1$ processes at the order of $\mathcal{O}\left(\lambda^{2}\right)$ and satisfying the global symmetry in eq. (2.3). Here, $\alpha$ and $\beta$ are QCD indices. The flavor indices are contracted inside the parenthesis. The operators above in the left panel are Hermitian operators, while the operators in the right panel including $\mathcal{O}_{V 1}, \mathcal{O}_{V 2}, \mathcal{O}_{S 1}, \mathcal{O}_{S 2}$ are all complex and can have $C P$ violating coefficients.

table $1{ }^{1}$ None of these operators generate $\Delta F=2$ observables at leading order, but, in the models considered below, such observables will be generated at one-loop. We will find that constraints from $\Delta F=2$ processes are nevertheless weak. For completeness, we also include the four-fermion operators containing leptons and at least one up-type quark. In this case, the MFV structure is analogous to that discussed above with the replacement $V \rightarrow U$, where $U$ is the Pontecorvo-Maki-Nakagawa-Sakata (PMNS) [22, 23] matrix.

The operators in the left panel of table 1 are Hermitian. They cannot yield new $C P$ violating phases and can only violate $C P$ via the CKM phase. The operators on the right panel, on the other hand, may have coefficients containing new $C P$ violating phases. This is unsurprising, since the matrix $\lambda_{D}$ may have a different overall phase from the matrix $\lambda_{U}$. The operators $\mathcal{O}_{V 1}$ and $\mathcal{O}_{V 2}$ can be rewritten with a scalar Lorentz structure using a Fiertz transformation as

$$
\begin{aligned}
& \mathcal{O}_{V 1}=2 V_{i l}\left(\lambda_{D}^{\dagger} V^{\dagger} \lambda_{U}\right)_{k j}\left(\bar{u}_{L \alpha}^{i} u_{R \beta}^{j}\right)\left(\bar{d}_{R \beta}^{k} d_{L \alpha}^{l}\right), \\
& \mathcal{O}_{V 2}=2 V_{i l}\left(\lambda_{D}^{\dagger} V^{\dagger} \lambda_{U}\right)_{k j}\left(\bar{u}_{L \alpha}^{i} u_{R \alpha}^{j}\right)\left(\bar{d}_{R \beta}^{k} d_{L \beta}^{l}\right),
\end{aligned}
$$

\footnotetext{
${ }^{1}$ We neglect operators constructed of $\sigma^{\mu \nu}$, which either have zero hadronic matrix elements for the $D$ meson decays in the naive factorization approximation or can be related to scalar operators via a Fierz transformation.
} 
Similarly, the operators $\mathcal{O}_{S 1}$ and $\mathcal{O}_{S 2}$ can be rewritten as

$$
\begin{aligned}
\mathcal{O}_{S 1} & =\frac{1}{2}\left(\lambda_{U}^{\dagger} V\right)_{i l}\left(\lambda_{D}^{\dagger} V^{\dagger}\right)_{k j}\left(\bar{u}_{R \alpha}^{i} u_{L \beta}^{j}\right)\left(\bar{d}_{R \beta}^{k} d_{L \alpha}^{l}\right)+\cdots, \\
\mathcal{O}_{S 2} & =\frac{1}{2}\left(\lambda_{U}^{\dagger} V\right)_{i l}\left(\lambda_{D}^{\dagger} V^{\dagger}\right)_{k j}\left(\bar{u}_{R \alpha}^{i} u_{L \alpha}^{j}\right)\left(\bar{d}_{R \beta}^{k} d_{L \beta}^{l}\right)+\cdots,
\end{aligned}
$$

where, we have not written the tensor operators containing $\sigma^{\mu \nu}$. We introduce neutral scalars to UV complete the operators $\mathcal{O}_{V 2}$ and $\mathcal{O}_{S 2}$ toward the end of this section.

The remainder of this paper concentrates on the two $C P$-violating operators, $\mathcal{O}_{V 2}$ and $\mathcal{O}_{S 2}$, since these operators are the only ones with a different color structure compared to the SM that can contain a new $C P$ violating phase under the assumption of MFV. They correspond to the electroweak-invariant operators:

$$
\begin{aligned}
& \mathcal{O}_{V 2}^{\mathrm{EW}}=2 V_{i l}\left(\lambda_{D}^{\dagger} V^{\dagger} \lambda_{U}\right)_{k j}\left(\tilde{H} \bar{Q}_{L \alpha}^{i} u_{R \alpha}^{j}\right)\left(\bar{d}_{R \beta}^{k} Q_{L \beta}^{l} H^{\dagger}\right), \\
& \mathcal{O}_{S 2}^{\mathrm{EW}}=\frac{1}{2}\left(\lambda_{U}^{\dagger} V\right)_{i l}\left(\lambda_{D}^{\dagger} V^{\dagger}\right)_{k j}\left(\bar{u}_{R \alpha}^{i} Q_{L \alpha}^{j}\right)\left(\bar{d}_{R \beta}^{k} Q_{L \beta}^{l}\right),
\end{aligned}
$$

where the $\mathrm{SU}(2)_{L}$ indices are contracted in the parenthesis for the dimension 8 operator $\mathcal{O}_{V 2}^{\mathrm{EW}}$ and between the two $Q_{L}$ 's for the dimension 6 operator $\mathcal{O}_{S 2}^{\mathrm{EW}}$.

\subsection{Phenomenology of the effective operators}

We now outline the most relevant $\Delta C=1$ and $\Delta T=1$ processes and perform some preliminary calculations of new physics contributions using the effective operators $\mathcal{O}_{V 2}$ and $\mathcal{O}_{S 2}$. These contributions are, as will be explored in sections 3 and 4 , the most significant ones. Keeping only the leading terms in the Lagrangian, we have

$$
\begin{array}{rlrl}
\mathcal{O}_{V 2}: & \Delta C=1: & 2 \lambda_{s} \lambda_{c} V_{12} V_{22}^{*}\left(\bar{u}_{L \alpha} c_{R \alpha}\right)\left(\bar{s}_{R \beta} s_{L \beta}\right), \\
\Delta T=1: & 2 \lambda_{b} \lambda_{t} V_{11} V_{33}^{*}\left(\bar{u}_{L \alpha} t_{R \alpha}\right)\left(\bar{b}_{R \beta} d_{L \beta}\right), \\
\mathcal{O}_{S 2}: & \Delta C=1: & \frac{1}{2} \lambda_{s} \lambda_{c} V_{22} V_{12}^{*}\left(\bar{c}_{L \alpha} u_{R \alpha}\right)\left(\bar{s}_{R \beta} s_{L \beta}\right), \\
\Delta T=1: & \frac{1}{2} \lambda_{b} \lambda_{t} V_{32} V_{33}^{*}\left(\bar{t}_{L \alpha} c_{R \alpha}\right)\left(\bar{b}_{R \beta} b_{L \beta}\right) .
\end{array}
$$

Other terms such as $2 \lambda_{b} \lambda_{t} V_{31} V_{13}^{*}\left(\bar{t}_{L \alpha} u_{R \alpha}\right)\left(\bar{b}_{R \beta} d_{L \beta}\right)$ for the $\Delta T=1$ processes from $\mathcal{O}_{S 2}$ have additional Cabbibo suppression and do not contribute significantly to the relevant processes. From eqs. $(2.11-2.14)$, one can see that for a same size contribution to $\Delta C=1$ processes, the operator $\mathcal{O}_{V 2}$ has a much larger contribution to $\Delta T=1$ processes than the operator $\mathcal{O}_{S 2}$.

We first consider contributions to $D$ meson direct $C P$ violation from these $\Delta C=1$ operators. At this point, we only perform estimates in an attempt to determine the scale required for there to be experimentally accessible contributions. A more complete analysis is performed in section 4 . For the decay channel $D^{0}\left(\bar{D}^{0}\right) \rightarrow K^{+} K^{-}$, one can define the following direct $C P$ violating observable

$$
A_{C P}^{K}=\frac{\Gamma\left(D^{0} \rightarrow K^{+} K^{-}\right)-\Gamma\left(\bar{D}^{0} \rightarrow K^{+} K^{-}\right)}{\Gamma\left(D^{0} \rightarrow K^{+} K^{-}\right)+\Gamma\left(\bar{D}^{0} \rightarrow K^{+} K^{-}\right)} .
$$


Assuming maximal $C P$ violating strong and weak phases, the contribution from $\mathcal{O}_{V 2}$ is estimated to be

$$
A_{C P}^{K} \sim \frac{4 \sqrt{2} \lambda_{c} \lambda_{s}}{N_{c} G_{F}} \frac{1}{\left|\Lambda^{2}\right|} \approx 0.002 \times\left(\frac{10 \mathrm{GeV}}{|\Lambda|}\right)^{2},
$$

where $|\Lambda|$ is the magnitude of the cutoff of the operator and the Yukawa coupling values of $y_{s}$ and $y_{c}$ are evaluated at the scale of $M_{Z}$ [24]. A similar estimate can be done for the asymmetry, $A_{C P}^{\pi}$, involving the decay $D^{0}\left(\bar{D}^{0}\right) \rightarrow \pi^{+} \pi^{-}$, which is highly suppressed by the $d$-quark Yukawa coupling. The cutoff must be $\mathcal{O}(10 \mathrm{GeV})$ to generate $A_{C P}^{K}$ close to the current experimental sensitivity.

We now perform some preliminary calculations of the most relevant top quark observable, the single top quark production cross-section. The new physics contributions to this observable due to the quark-level process $u \bar{d} \rightarrow t \bar{b}$ are calculated by assuming a scalar particle with a mass $\Lambda$ propagates in a $t$-channel diagram with couplings to $u \bar{t}$ and $d \bar{b}$ denoted by $\bar{\kappa}_{U}$ and $\bar{\kappa}_{D}$. There is no CKM suppression for these couplings, but they are proportional to appropriate Yukawa couplings. The Yukawa couplings are, in turn, sensitive to $\tan \beta$ in two Higgs doublet models. With some $\tan \beta$ enhancement, both couplings $\bar{\kappa}_{U}$ and $\bar{\kappa}_{D}$ can be $\mathcal{O}(1)$. Choosing $\bar{\kappa}_{U}=\bar{\kappa}_{D}=1$ and the scalar particle mass to be $10 \mathrm{GeV}$, we have new physics contributions to single top production given by

$$
\begin{aligned}
\sigma[p \bar{p} \rightarrow t \bar{b}(b \bar{t})] & =0.11 \mathrm{pb} & & (1.96 \mathrm{TeV} \text { Tevatron }), \\
\sigma[p p \rightarrow t \bar{b}(b \bar{t})] & =0.56 \mathrm{pb} & & (8 \mathrm{TeV} \text { LHC })
\end{aligned}
$$

Since the contribution to $D$ meson $C P$ violation is linear in the product of couplings $\bar{\kappa}_{U} \bar{\kappa}_{D}$, while top production cross sections are quadratic in the product of couplings $\bar{\kappa}_{U}^{2} \bar{\kappa}_{D}^{2}$, increasing both the mediator mass and couplings simultaneously keeps contributions to $D$ meson $C P$ violation fixed while dramatically enhancing single top production.

Similarly, for the other operator $\mathcal{O}_{S 2}$, we have

$$
A_{C P}^{K} \sim \frac{\sqrt{2} \lambda_{c} \lambda_{s}}{N_{c} G_{F}} \frac{\chi_{K}}{8 N_{c}} \frac{1}{\Lambda^{2}} \approx 0.001 \times\left(\frac{5 \mathrm{GeV}}{\Lambda}\right)^{2}
$$

where the chiral factor $\chi_{K} \approx 2 m_{K}^{2} /\left(m_{c} m_{s}\right) \approx 4.2$ for $m_{c}$ and $m_{s}$ evaluated at the $D$ meson mass. The single top production cross sections are suppressed by the CKM element $\left|V_{32}\right|^{2}$. For $\bar{\kappa}_{U}=\bar{\kappa}_{D}=1$ and scalar particle mass $10 \mathrm{GeV}$, they are

$$
\begin{aligned}
\sigma[p \bar{p} \rightarrow t \bar{b}(b \bar{t})] & =5.1 \times 10^{-8} \mathrm{pb} & & (1.96 \mathrm{TeV} \text { Tevatron }) \\
\sigma[p p \rightarrow t \bar{b}(b \bar{t})] & =3.1 \times 10^{-6} \mathrm{pb} & & (8 \mathrm{TeV} \mathrm{LHC})
\end{aligned}
$$

This operator has a negligible new physics contribution to the single top production cross section if there is a sub-percent level contribution to the $D$ meson $C P$ violation. From these estimates for $\mathcal{O}_{V 2}$ and $\mathcal{O}_{S 2}$, one can already see that different structures of the effective operators that give contributions to $C P$ violation in $D$-meson decays have dramatically different predictions for top quark physics. 


\begin{tabular}{|ccc|}
\hline Model \# & $\phi$ flavor & Operators Generated \\
\hline 1 & $(1,3,1, \overline{3})$ & $\mathcal{O}_{S 2}, \mathcal{O}_{V 2}$ \\
2 & $(1,3, \overline{3}, 1)$ & $\mathcal{O}_{S 2}, \mathcal{O}_{V 2}$ \\
3 & $(1,1,3, \overline{3})$ & $\mathcal{O}_{S 2}$ \\
4 & $(1,8,1,1)$ & $\mathcal{O}_{S 2}, \mathcal{O}_{V 2}$ \\
5 & $(1, \overline{3}, \overline{3}, \overline{3})$ & $\mathcal{O}_{V 2}$ \\
6 & $(1,8,3, \overline{3})$ & $\mathcal{O}_{S 2}$ \\
7 & $(1,6, \overline{3}, \overline{3})$ & $\mathcal{O}_{V 2}$ \\
\hline
\end{tabular}

Table 2. Scalar flavor structures under SU(3) ${u_{L}}_{L} \times \mathrm{SU}(3)_{d_{L}} \times \mathrm{SU}(3)_{u_{R}} \times \mathrm{SU}(3)_{d_{R}}$ that are allowed by requiring a neutral scalar that reproduces the flavor structure of the $C P$-violating operators.

\subsection{Light mediators}

Because of the Yukawa coupling suppression in MFV, the new physics affects on $D$ meson $C P$ violation and top quark properties are typically small for $\Lambda \gtrsim 100 \mathrm{GeV}$. On the other hand, if the new particle inducing the MFV operator is lighter than $100 \mathrm{GeV}$, then there can be large effects. We do not consider masses below $\mathcal{O}(10 \mathrm{GeV})$ because of potentially severe constraints from decays of bottom-quark bound states and searches for light hadronic resonances in fixed-target experiments [25]. For this range of scales, $\mathcal{O}(10 \mathrm{GeV})<\Lambda<\mathcal{O}(100 \mathrm{GeV}), D$ meson $C P$ violation effects can still be calculated in an effective operator approach. For the top quark physics, however, the actual new physics degree of freedom enters both production and decay. We therefore pay special attention to the case in which the MFV operator is generated by a light particle. Because of various collider constraints, new light particles with a mass below $100 \mathrm{GeV}$ must be neutral under SM gauge interactions. The particle must be a boson in order to generate the operators $\mathcal{O}_{V 2}$ and $\mathcal{O}_{S 2}$. We introduce a new scalar gauge singlet $\phi$ which transforms under flavor as a completion for these operators between the $10-100 \mathrm{GeV}$ scales and study the class of such scalars.

There are several possible flavor representations for the scalar $\phi$. We list the flavor symmetry possibilities in table 2 . Note that fields $\phi$ transforming under $\mathrm{SU}(3)_{u_{L}}$ and under $\mathrm{SU}(3)_{d_{L}}$ are equivalent up to a basis change since, in either case, the representation under $G_{F}^{\mathrm{EW}}$ arises from a representation under $G_{F}^{\mathrm{EW}}$. We therefore choose, without loss of generality, to consider only cases where $\phi$ transforms under $\mathrm{SU}(3)_{d_{L}}$. Some representations can only generate one operator, while other representations can generate two operators. We will use the representation $(1,3,1, \overline{3})$ as a prototype for our phenomenological studies, as this representation contributes to both $C P$ violation in $D$ meson decays and processes involving top quarks, capturing the full breadth of potential effects due to a light scalar. At the order of magnitude level, contributions to a given operator due to the other representations are comparable. The leading couplings for a scalar with this flavor representation 
are

$$
\begin{aligned}
\mathcal{L} \supset \kappa_{U_{L}} \bar{u}_{R}^{i}\left(\lambda_{U}^{\dagger} V\right)^{i l} \phi_{l k}\left(\lambda_{D}^{\dagger} V^{\dagger}\right)^{k j} u_{L}^{j}+\kappa_{U_{R}} \bar{u}_{L}^{i} V^{i l} \phi_{l k}\left(\lambda_{D}^{\dagger} V^{\dagger} \lambda_{U}\right)^{k j} u_{R}^{j}+\text { h.c. } \\
\quad+\kappa_{D} \bar{d}_{R}^{k}\left(\phi^{\dagger}\right)_{k l} d_{L}^{l}+\text { h.c. }+m_{\phi}^{2} \phi^{\dagger} \phi,
\end{aligned}
$$

where we neglect additional scalar potential terms as well as additional mass terms that split the $\phi_{l k}$ components. Perturbativity limits are saturated when $\kappa_{D} \sim \sqrt{4 \pi}$ or $\kappa_{U_{L}}, \kappa_{U_{R}} \sim 200$ (the largest coupling is proportional to $\lambda_{b}$ and $\lambda_{t}$ ). Under the assumption that the potential for $\phi$ conserves flavor, the interactions eq. (2.22) break the global U(1) under which only $\phi$ transforms. Redefinitions of $\phi$ then give the freedom to remove the phase of one of the three couplings. For concreteness and without loss of generality, we work in a basis where $\kappa_{D}$ is real and the couplings $\kappa_{U_{L, R}}$ are complex. Integrating out $\phi$, one can generate both $\mathcal{O}_{V 2}$ and $\mathcal{O}_{S 2}$ operators with low energy Lagrangian

$$
\mathcal{L}=\frac{\kappa_{U_{R}} \kappa_{D}}{2 m_{\phi}^{2}} \mathcal{O}_{V 2}+\frac{\kappa_{U_{L}} \kappa_{D}}{2 m_{\phi}^{2}} \mathcal{O}_{S 2}+\text { h.c. }
$$

In the following sections, we elaborate on the phenomenology of this model.

\section{Top quark properties}

A light $\phi$ with interactions given in eq. (2.22) would modify top production and decay. From this equation, the leading couplings mediating $\Delta T=1$ processes are

$$
\begin{aligned}
\mathcal{L} \supset & \kappa_{U_{L}} \lambda_{b} \lambda_{t}\left(\bar{t}_{R} \phi_{33} V_{33}^{*} V_{23}^{*} c_{L}+\bar{t}_{R} \phi_{33} V_{33}^{*} V_{13}^{*} u_{L}\right) \\
& +\kappa_{U_{R}} \lambda_{b} \lambda_{t}\left(\bar{u}_{L} \phi_{13} V_{11} V_{33}^{*} t_{R}+\bar{c}_{L} \phi_{23} V_{22} V_{33}^{*} t_{R}\right)+\text { h.c. } \\
= & \bar{\kappa}_{U_{L}}\left(\bar{t}_{R} \phi_{33} V_{33}^{*} V_{23}^{*} c_{L}+\bar{t}_{R} \phi_{33} V_{33}^{*} V_{13}^{*} u_{L}\right) \\
& +\bar{\kappa}_{U_{R}}\left(\bar{u}_{L} \phi_{13} V_{11} V_{33}^{*} t_{R}+\bar{c}_{L} \phi_{23} V_{22} V_{33}^{*} t_{R}\right)+\text { h.c. }
\end{aligned}
$$

where, for convenience, we have defined $\bar{\kappa}_{U_{L}} \equiv \kappa_{U_{L}} \lambda_{b} \lambda_{t}$ and $\bar{\kappa}_{U_{R}} \equiv \kappa_{U_{R}} \lambda_{b} \lambda_{t}$. The coupling $\bar{\kappa}_{U_{L}}$ has an additional CKM angle suppression such that, for perturbative couplings, there is negligible effect on top physics. We therefore focus on contributions from $\bar{\kappa}_{U_{R}}$.

\subsection{Single top production}

In this model, there are two additional contributions to single top production. The first is $u \bar{d} \rightarrow t \bar{b}$ with $\phi_{13}$ being exchanged in the $t$-channel and the second is $u g \rightarrow t \phi_{13}$ for which there are two tree level diagrams. For the first channel, neglecting the $b$-quark mass in the final state, there is no interference terms between the $W$ mediated and the $\phi_{13}$ mediated diagrams. The leading order contribution to the parton-level cross section is

$$
\sigma(u \bar{d} \rightarrow t \bar{b})=\frac{\left|\bar{\kappa}_{U_{R}}\right|^{2}\left|\kappa_{D}\right|^{2}}{16 \pi s^{2}}\left[\left(2 m_{\phi}^{2}-m_{t}^{2}\right) \ln \left(\frac{m_{\phi}^{2}}{s+m_{\phi}^{2}-m_{t}^{2}}\right)+\frac{\left(s-m_{t}^{2}\right)\left(s+2 m_{\phi}^{2}-2 m_{t}^{2}\right)}{s+m_{\phi}^{2}-m_{t}^{2}}\right],
$$

where $\sqrt{s}$ is the parton center-of-mass energy. There are two diagrams contributing to the production of $u g \rightarrow t \phi_{13}$ : one is from exchanging a $t$ quark in the $t$-channel and other is 

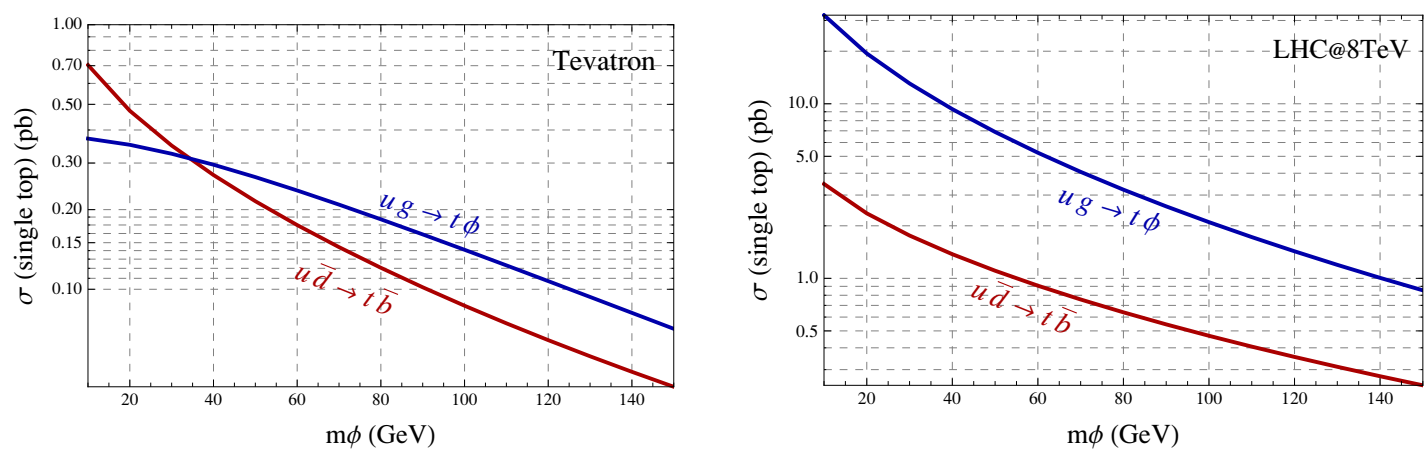

Figure 1. The single top production cross sections from the contributions of the new neutral scalar $\phi$. The couplings are chosen to be $\left|\bar{\kappa}_{U_{R}}\right|=\left|\kappa_{D}\right|=0.2$.

from exchanging a $u$ quark in the $s$-channel. Neglecting the $\phi$ particle mass, the leading order parton-level cross-section is

$$
\sigma\left(u g \rightarrow t \phi_{13}\right)=\frac{g_{s}^{2}\left|\bar{\kappa}_{U_{R}}\right|^{2}}{192 \pi s^{3}}\left[2\left(s^{2}+2 s m_{t}^{2}+2 m_{t}^{4}\right) \ln \left(\frac{s}{m_{t}^{2}}\right)+7 m_{t}^{4}-4 s m_{t}^{2}-3 s^{2}\right] .
$$

The production cross sections at the Tevatron and $8 \mathrm{TeV}$ LHC as a function of $m_{\phi}$ are shown in figure 1. We use the Mathematica MSTW 2008 PDFs [26]. Production from $u$ and $g$ partons dominates at the $8 \mathrm{TeV}$ LHC. The latest measurement of single top production from the CDF collaboration at Tevatron with $7.5 \mathrm{fb}^{-1}$ has an error below $1 \mathrm{pb}$ [27], while the latest result from the CMS collaboration with $5.0 \mathrm{fb}^{-1}$ at the $8 \mathrm{TeV} \mathrm{LHC}$ has an error around $10 \mathrm{pb}$ [28]. We will use these experimental results to constrain the $\phi$ parameter space in section 4 .

\section{$3.2 t \bar{t}$ pair-production}

We first comment on same-sign $t t$ production for this model. Neglecting the coupling $\kappa_{D}$, $t$-number global symmetry is only broken by the CKM elements and $t t$ production is suppressed. Including the coupling $\kappa_{D}$, this model generates same-sign top final state without CKM suppression, but with a large final state multiplicity, for instance $u u \rightarrow t t \bar{b} \bar{b} d d$. The cross section is then strongly suppressed by phase space. We therefore concentrate on $t \bar{t}$ production.

The dominant new physics contribution to $t \bar{t}$ pair-production is through exchange of the $\phi$ field in the $t$-channel. In addition, the interference with SM gluon exchange in the $s$ channel cannot be neglected. The leading order parton-level pair production cross-section, neglecting the mass of $\phi$, is given by

$$
\begin{aligned}
\sigma(u \bar{u} \rightarrow t \bar{t})= & \frac{1}{216 \pi s}\left\{\beta\left[g_{s}^{4}\left(8 m^{2}+4\right)-6 g_{s}^{2}\left(2 m^{2}+1\right)\left|\bar{\kappa}_{U_{R}}\right|^{2}+27\left|\bar{\kappa}_{U_{R}}\right|^{4}\right]\right. \\
& \left.-3 m^{2}\left|\bar{\kappa}_{U_{R}}\right|^{2} \ln \left(\frac{1+\beta-2 m^{2}}{1-\beta-2 m^{2}}\right)\left[4 g_{s}^{2}\left(m^{2}+1\right)-9\left|\bar{\kappa}_{U_{R}}\right|^{2}\right]\right\}
\end{aligned}
$$

where $m^{2} \equiv m_{t}^{2} / s$ and $\beta=\sqrt{1-4 m^{2}}$. Figure 2 shows the modifications on the $t \bar{t}$ produc- 


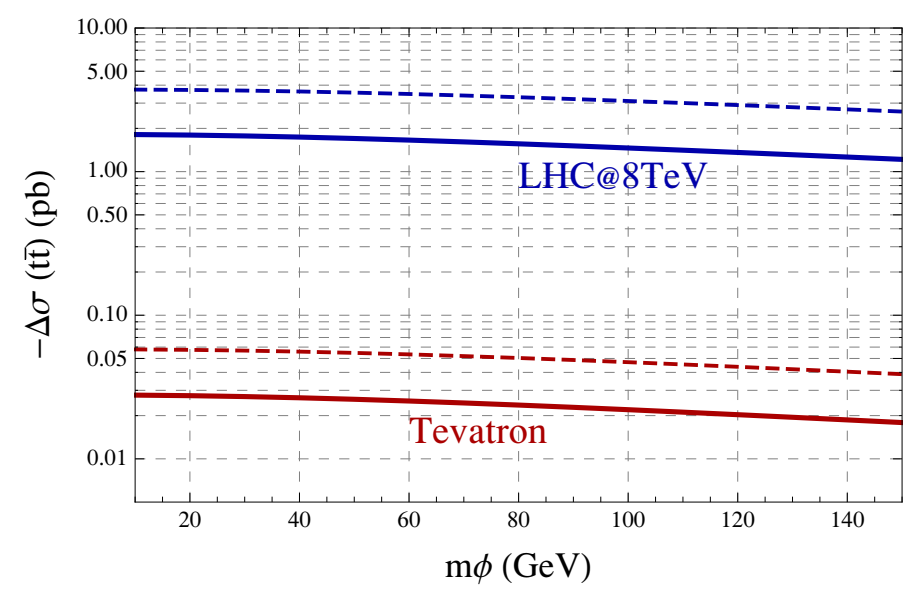

Figure 2. Modification of the $t \bar{t}$ production cross sections from contributions of the new neutral scalar $\phi$. The couplings are chosen to be $\left|\bar{\kappa}_{U_{R}}\right|=0.2$ for the two solid lines and $\left|\bar{\kappa}_{U_{R}}\right|=0.3$ for the two dashed lines.

tion cross section. Because of the deconstructive interference (taking $\left|\bar{\kappa}_{U_{R}}\right|=0.2$ or 0.3 ), the new $\phi$ contribution decreases the total production cross section by a few pb at the $8 \mathrm{TeV}$ LHC and tens of $\mathrm{fb}$ at the Tevatron. The current uncertainty on the $t \bar{t}$ pair-production cross section at the $8 \mathrm{TeV}$ LHC is approximately $30 \mathrm{pb}$ [29] from the ATLAS collaboration and $0.4 \mathrm{pb}$ at Tevatron [30], so the modifications on $t \bar{t}$ pair-production do not significantly constrain the parameter space of this model.

\subsection{Non-standard top decays}

For a light $\phi$ scalar with $m_{\phi}<m_{t}$, the top quark can decay into $\phi$ plus the up quark or charm quark. Since the CKM matrix is nearly diagonal, top quark decay yields primarily $\phi_{13}\left(\phi_{23}\right)$ for an up (charm) and $\phi$ final state. A $\phi$ with such flavor indices decays exclusively to a $b$ quark and a light quark. For $m_{\phi} \lesssim 20 \mathrm{GeV}$, the two resulting jets are collimated and behave as a fat jet, so the top quark is observed as a dijet resonance. For a heavier $\phi$, the top quark looks like a three-jet resonance. Summing the two dominant new decay channels, $t \rightarrow u+\phi_{13}$ and $t \rightarrow c+\phi_{23}$, the partial width for this mode is

$$
\Gamma(t \rightarrow j+\phi)=\frac{\left|\bar{\kappa}_{U_{R}}\right|^{2}}{8 \pi} m_{t}\left(1-\frac{m_{\phi}^{2}}{m_{t}^{2}}\right) .
$$

Using the latest theoretical results for the top quark decay width in the SM [31-34], we show the branching ratio of the new decay channel in figure 3. The current experimental value of the top quark width is $\Gamma_{t}=1.99_{-0.55}^{+0.69} \mathrm{GeV}$ from D0 [35], which is extracted using the partial decay width $\Gamma(t \rightarrow W b)$ measured from the $t$-channel cross section for single top quark production and from the branching fraction of $\operatorname{Br}(t \rightarrow W b)$ measured in $t \bar{t}$ events. As we will see below, the total top quark width measurement does constrain the parameter space for $\phi$.

The $\phi$ can also introduce additional contributions to the top quark forward-backward or charge asymmetries. For the parameter space allowed by the single top production cross section, however, the modifications on the top quark pair production are negligible, and 


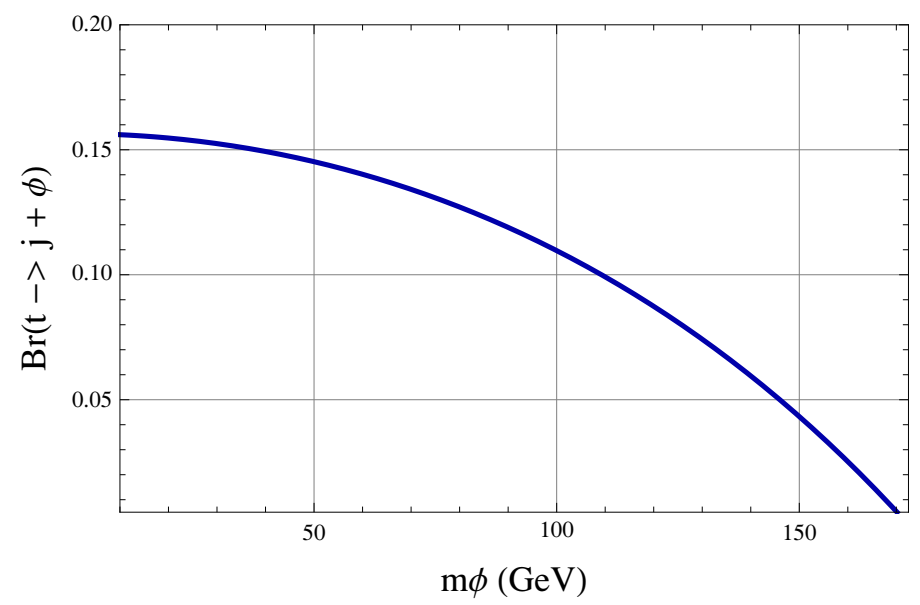

Figure 3. The branching ratio of the new top quark decay channel. The coupling is chosen to be $\left|\bar{\kappa}_{U_{R}}\right|=0.2$. For a light $\phi$ field, the top quark may behave as a dijet at colliders.

hence the amount of asymmetry is unlikely to be visible at hadron colliders. A lepton collider such as the International Linear Collider [36-39] would be an ideal machine to probe this model's parameter space.

\section{Additional constraints and D-meson physics}

Both $b$-quark and $c$-quark physics may be modified by the introduction of the new light scalar. As mentioned in the introduction, we are interested in the up-type quark sector of MFV models. The main focus of our work is therefore on $\Delta T=1$ and $\Delta C=1$ processes. On the other hand, the MFV mediator, $\phi$, also couples to down-type quarks, modifying their properties. We study the most accessible $b$-quark and $c$-quark physics in this section.

\subsection{Enhanced $b$ production}

The interactions introduced in eq. (2.22) yield unsuppressed couplings of $\phi$ to the downtype sector. Fortunately, the coupling $\kappa_{D}$ does not mediate flavor and does not allow for flavor violation without coupling to the up sector, so many processes, including $\Delta F=2$ FCNCs are not induced. There are subdominant bounds at low masses $m_{\phi} \lesssim 10 \mathrm{GeV}$ from fixed-target searches for hadronic resonances [25]. For the masses $m_{\phi}>10 \mathrm{GeV}$ that we are studying, the most stringent bounds come from $Z$ decays via a process illustrated in figure 4. Existing LEP searches are most sensitive to three body decays of the form $Z \rightarrow q \bar{q}^{\prime} \phi$. In particular, $Z \rightarrow b \bar{b} \phi$ is severely constrained from $Z \rightarrow b \bar{b} b \bar{b}$ searches [40]. These searches require only three $b$-jets, under the assumption that the fourth will be present in all cases. A $\phi_{33}$ would either look exactly like a $b$-jet at low mass or would decay dominantly to two $b$-jets at higher masses. ${ }^{2}$ Both final states would be accepted by the analysis cuts for the $Z \rightarrow b \bar{b} b \bar{b}$ measurement.

\footnotetext{
${ }^{2}$ The LEP experiments used a jet definition based on a cut on $y=M_{i j}^{2} / s$. Typical values of $y_{\min }$ are few $\times 10^{-2}$, corresponding to $M_{i j} \sim 20 \mathrm{GeV}$. Using this definition, a relatively light $\phi$ would appear as a single jet to the experiment.
} 

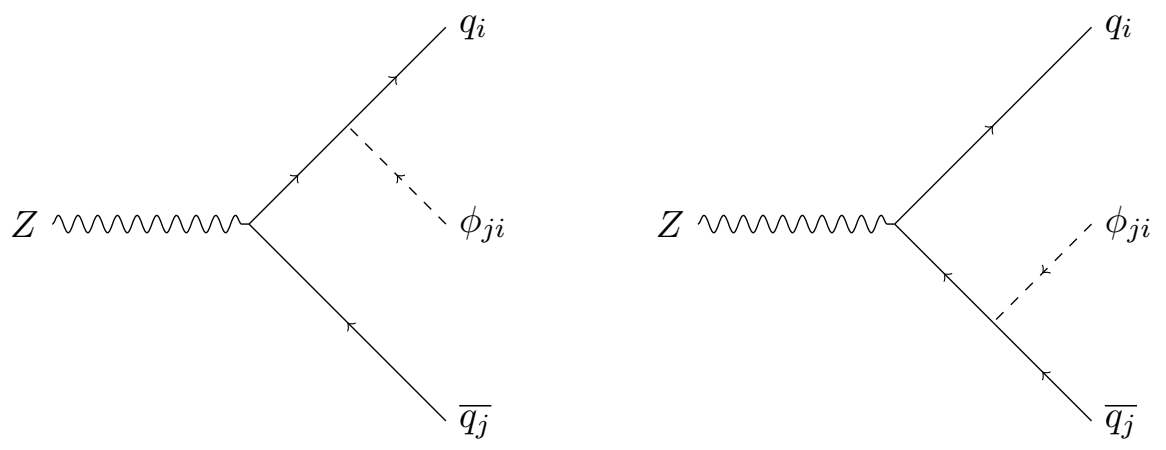

Figure 4. Diagrams for the decay $Z \rightarrow q_{i} \overline{q_{j}} \phi_{j i}$.

To work out the bounds in detail, we also need to know the properties of the $\phi$ field, in particular the branching ratio for its decay into two $b$-jets. Because the coupling $\kappa_{D}$ does not violate flavor, the final state from $Z \rightarrow q \bar{q}^{\prime} \phi$ should contain an even number of $b$-jets. Since only the decay with $\phi_{33}$ can give $4 b$ final states, we only need to consider $Z \rightarrow q \bar{q}^{\prime} \phi_{33}$, where the $\phi_{33}$ field mainly decays into two $b$-jets. Other $\phi_{33}$ decay channels via its couplings to up-type quarks are suppressed both by CKM angles and Yukawa couplings; they can be neglected. We therefore assume $100 \%$ branching ratio for $\phi_{33} \rightarrow b \bar{b}$.

To get a better idea of the constraints, we calculate the partial width of the general processes $Z \rightarrow q_{i} \bar{q}_{j} \phi_{j i}$. The rate $Z \rightarrow \bar{q}_{i} q_{j} \phi_{j i}^{*}$ is the same by $C P$. The details of our calculation of the partial width are given in appendix A. The integration over phase space was performed numerically including all quark masses and the $\phi$ mass. Nevertheless, it is instructive to examine an approximate expression for the partial width in the limit $m_{b}, m_{\phi} \ll m_{Z}$

$$
\Gamma\left(Z \rightarrow q_{i} \bar{q}_{j} \phi_{j i}\right) \sim \frac{\alpha \kappa_{D}^{2} m_{Z}}{576 \pi^{2} s_{w}^{2} c_{w}^{2}}=(0.35 \mathrm{MeV}) \times \kappa_{D}^{2} .
$$

This corresponds to a branching fraction of order $10^{-4}$ for $\kappa_{D}$ of order 1 , which, as we see below, is close to the current sensitivity.

As mentioned above, the most severe constraint arises from LEP searches for $Z \rightarrow b \bar{b} b \bar{b}$. The rate for this process is measured by both the OPAL and DELPHI collaborations [41, 42] with branching ratio

$$
\mathrm{Br}_{\exp }(Z \rightarrow b \bar{b} b \bar{b})=(3.6 \pm 1.3) \times 10^{-4} .
$$

The total $Z$ width has been measured as $\Gamma_{\mathrm{SM}}=2.4952 \pm 0.0023 \mathrm{GeV}$ [40]. The branching ratio from the new physics with $m_{\phi}=15 \mathrm{GeV}$ and $\left|\kappa_{D}\right|=1$ is

$$
\operatorname{Br}\left(Z \rightarrow b \bar{b} \phi_{33} \rightarrow 2 b 2 \bar{b}\right)+\operatorname{Br}\left(Z \rightarrow b \bar{b} \phi_{33}^{*} \rightarrow 2 b 2 \bar{b}\right)=2.9 \times 10^{-4} .
$$

Obtaining an accurate SM prediction for the $4 b$ branching fraction of the $Z$ is challenging because it is a QCD process with large logs. On the other hand, it is certain that the new physics contribution cannot exceed the upper limit on the total observed branching fraction. Using the $1 \sigma$ upper limit given above, $\mathrm{Br}_{\text {new }}(\mathrm{Z} \rightarrow \mathrm{b} \bar{b} b \bar{b})<4.9 \times 10^{-4}$, the constraint on the coupling for $m_{\phi}=15 \mathrm{GeV}$ is $\kappa_{D}<1.28$. The bound over the full range 


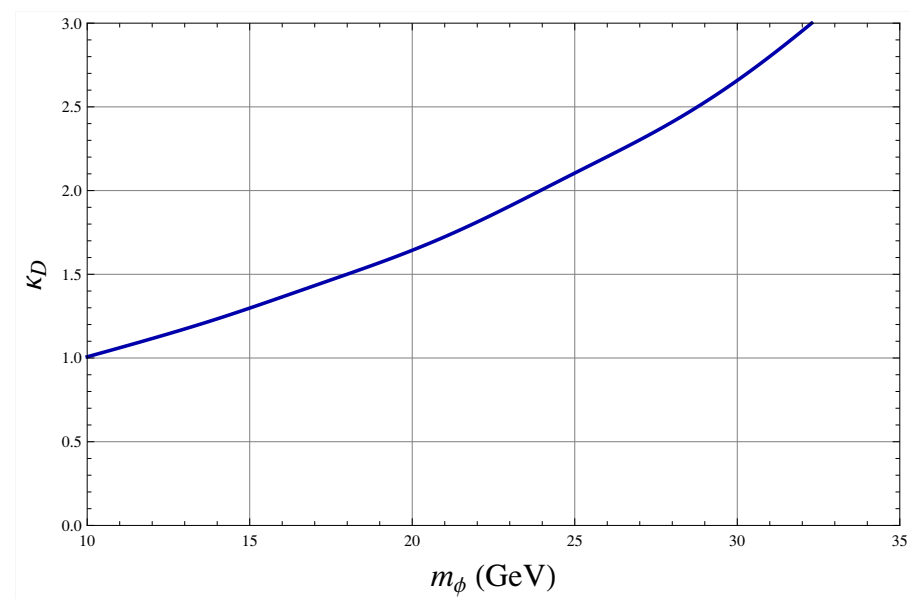

Figure 5. Upper limit on $\kappa_{D}$ from the measurement of $\operatorname{Br}(\mathrm{Z} \rightarrow \mathrm{b} \overline{\mathrm{b}} \overline{\mathrm{b}} \mathrm{b})$ as a function of $\phi$ particle masses.

of interesting $\phi$ masses is presented in figure 5. We can see from this figure that the limits on the coupling $\kappa_{D}$ reaches the non-perturbative range for a heavy $m_{\phi} \sim 30 \mathrm{GeV}$.

\section{2 $D$ meson direct $C P$ violation}

A striking consequence of a new light neutral scalar for charm physics is the possibility of generating a significant direct $C P$ asymmetry in $D$ meson decays. In some parts of parameter space, the asymmetry generated can be large enough to explain the anomaly observed by Belle, CDF, and $\mathrm{LHCb}$ without requiring an enhancement of "penguin" contributions relative to the naive expectation, as explored in ref. [43]. Other effects on charm physics are negligible as they are overwhelmed by SM contributions. For example, $D^{0}-\overline{D^{0}}$ mixing typically provides a very stringent bound for new physics contributions to the $\Delta F=2$ processes. With MFV implemented at tree-level, however, $D^{0}-\overline{D^{0}}$ mixing is generated only at one loop with bottom Yukawa suppression and does not constrain parameter space of this model.

Direct $C P$ violation in the $D$ system has long been lauded as a "smoking gun" signature of BSM physics [44]. Recent measurements of the direct $C P$ asymmetry difference between $D^{0} \rightarrow K^{+} K^{-}$and $D^{0} \rightarrow \pi^{+} \pi^{-}$may provide the first hint of such $C P$ violation. The observable is defined as

$$
\Delta A_{C P} \equiv A_{C P}^{K}-A_{C P}^{\pi}
$$

For the MFV models considered so far, we have $A_{C P}^{K} \gg A_{C P}^{\pi}$ and thus $\Delta A_{C P} \approx A_{C P}^{K}$ since the asymmetry for pions is Yukawa suppressed. To tag the $D^{0}$, one can use the $\pi^{+}$ from $D^{*+} \rightarrow D^{0}+\pi^{+}$or the muon from $B \rightarrow D \mu X$. For the $\pi^{+}$tagging, and adding the errors in quadrature, we obtain the value $\Delta A_{C P}=(-0.46 \pm 0.13) \%$ averaged over the results from BaBar [45], Belle [46], CDF [47] and LHCb [48]. For the muon tagging, the latest measurement from LHCb using $1.0 \mathrm{fb}^{-1}$ data at $7 \mathrm{TeV}$ has $\Delta A_{C P}=(0.49 \pm$ $0.30 \pm 0.14) \%$ [49], which has opposite sign compared to the $\pi$-tagged result. Combining 


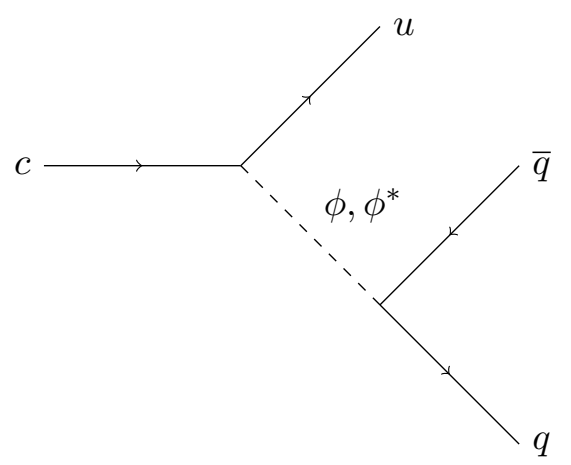

Figure 6. Diagram for the $D$ meson decay to $K K$ and $\pi \pi$ via the new scalar $\phi$. The $K K$ and $\pi \pi$ modes correspond to $q=s$ and $q=d$ respectively.

the results from both tagging channels, the current world-averaged direct $D$ meson $C P$ violation result is [50]:

$$
\Delta A_{C P}^{\exp }=(-0.329 \pm 0.121) \%,
$$

which corresponds to a $2.7 \sigma$ significance. The SM prediction for this quantity is estimated to be smaller than $\mathcal{O}\left(10^{-3}\right)[44]$.

In addition to the recent decrease in the significance of the observed $C P$ violation in $D$ decays, there has been renewed theoretical study of direct $C P$ violation in the $D$ system. An enhancement of the relevant hadronic matrix element, analogous to the $\Delta I=$ $\frac{1}{2}$ rule in Kaon physics (see ref. [51, 52] for recent Lattice calculations), may predict a larger value of $\Delta A_{C P}$, as pointed out in ref. [53,54]. Recent work has shown that the assumption of a large " $\Delta U=0$ rule" for $D$ decays, i.e. that $\Delta U=0$ amplitudes receive a factor of $\sim 10$ enhancement compared to $\Delta U=1$ amplitudes, can simultaneously explain several outstanding puzzles in $D$ physics [55]. Such an enhancement is larger than naively expected from QCD estimations, but an accurate calculation is beyond the reach of current techniques in the $D$ system.

We now explore the possibility that the MFV operators of $\mathcal{O}_{V 2}$ and $\mathcal{O}_{S 2}$ explain the observed value of $\Delta A_{C P}^{\text {exp }}$ in eq. (4.5). We assume that naive factorization holds so that the $\mathrm{SM}$ contribution is negligible. We integrate out $\phi$ and run the resulting operators down to the $D$ meson mass scale. The Feynman diagram for $D$ meson decay is illustrated in figure 6 . At the scale of the scalar $\phi$, we generate the operators

$$
\begin{aligned}
& \frac{\kappa_{U_{L}} \kappa_{D}}{m_{\phi}^{2}}\left(\lambda_{U}^{\dagger} V\right)_{i l}\left(\lambda_{D}^{\dagger} V^{\dagger}\right)_{k j}\left(\bar{u}_{R \alpha}^{i} u_{L \alpha}^{j}\right)\left(\bar{d}_{R \beta}^{k} d_{L \beta}^{l}\right)+\text { h.c. } \\
& +\frac{\kappa_{U_{R}} \kappa_{D}}{m_{\phi}^{2}} V_{i l}\left(\lambda_{D}^{\dagger} V^{\dagger} \lambda_{U}\right)_{k j}\left(\bar{u}_{L \alpha}^{i} u_{R \alpha}^{j}\right)\left(\bar{d}_{R \beta}^{k} d_{L \beta}^{l}\right)+\text { h.c. }
\end{aligned}
$$

From this equation, one can read off the Wilson coefficients:

$$
C_{V 2}\left(m_{\phi}\right)=\frac{1}{2 m_{\phi}^{2}} \kappa_{U_{R}} \kappa_{D}, \quad C_{S 2}\left(m_{\phi}\right)=\frac{1}{2 m_{\phi}^{2}} \kappa_{U_{L}} \kappa_{D}
$$



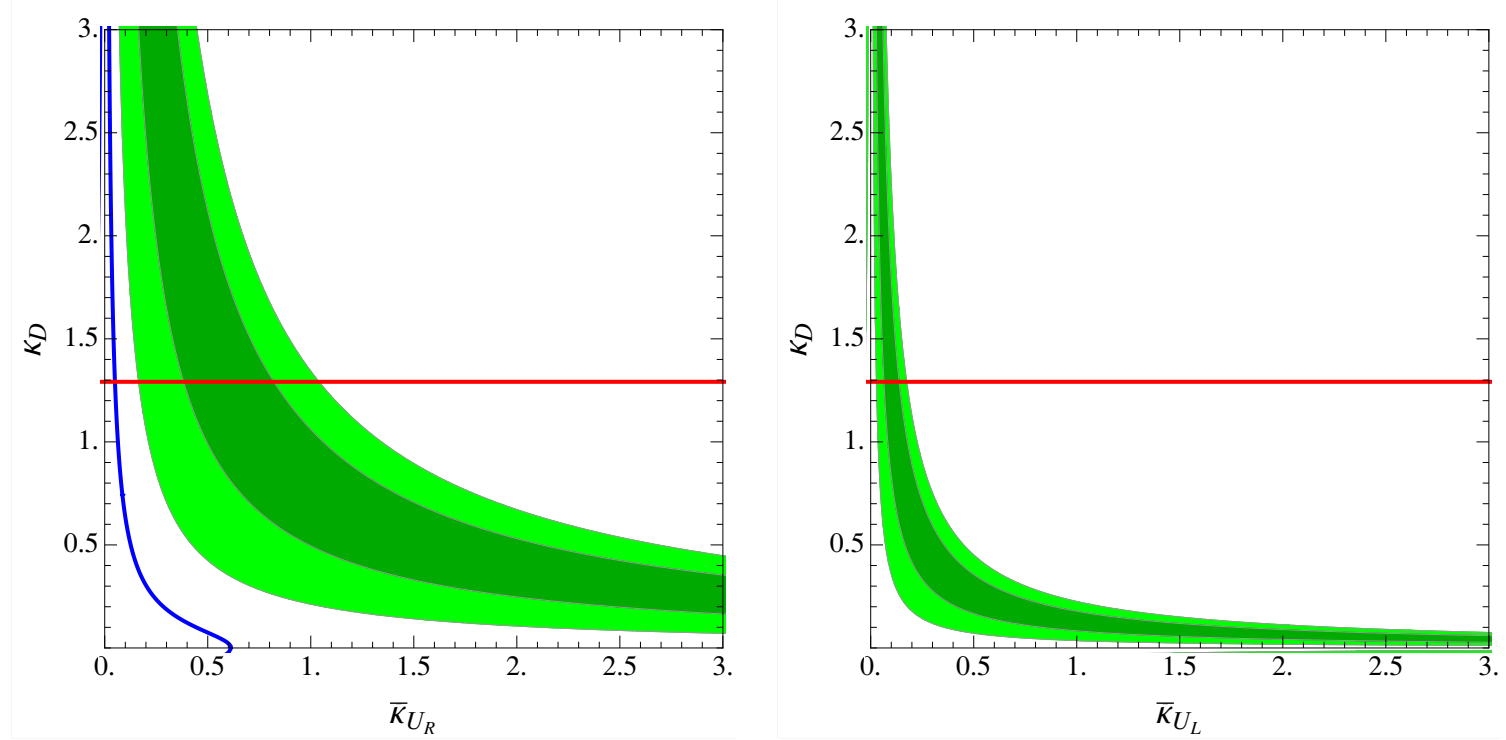

Figure 7. The $1 \sigma$ and $2 \sigma$ contour plots of the parameter space to accommodate the direct $C P$ violation variable $A_{C P}^{\exp }$ in the $D$-meson system. The neutral scalar mass is chosen to be $10 \mathrm{GeV}$. The red horizontal line is the upper limit on $\kappa_{D}$ from the measurement of $\operatorname{Br}(Z \rightarrow b \bar{b} b \bar{b})$. The blue curve in the left panel is the upper limit constrained from the single-top production cross section at CDF.

In addition to these operators, a tensor operator is generated, but it does not contribute to $C P$ violation as it has a zero matrix element assuming naive factorization [56]. The tensor operator does, however, give a significant contribution to the renormalization group running of the scalar coefficients. The details of this running are described in appendix B, while the estimation of the relevant hadronic matrix element ratios are performed in appendix C. In terms of the low-energy operator, the direct $C P$ asymmetry is given by

$$
A_{C P}^{K} \approx \frac{2 \sqrt{2}}{N_{c} G_{F}} \lambda_{c} \lambda_{s}\left\{\frac{1}{4} \sin \delta_{V 2} \operatorname{Im}\left[C_{V 2}\left(m_{D}\right)\right]-\frac{1}{8} \chi_{K} \sin \delta_{S 2} \operatorname{Im}\left[C_{S 2}\left(m_{D}\right)\right]\right\},
$$

where $\delta_{V 2}$ and $\delta_{S 2}$ are the strong phases of the matrix elements of $\mathcal{O}_{V 2}$ and $\mathcal{O}_{S 2}$. One has a similar expansion for $A_{C P}^{\pi}$ by replacing $\lambda_{s}$ by $\lambda_{d}$. The strong phases are estimated to be $\mathcal{O}(1)$ in QCD decays and we take them to have the maximal value: $\sin \delta_{V 2}=1$ and $\sin \delta_{S 2}=1$. We also assume a maximal weak phase for the coefficients of these operators: $\arg C_{V 2}=\pi / 2$ and $\arg C_{S 2}=\pi / 2$. We neglect subdominant effects from interference between $\mathcal{O}_{V 2}$ and $\mathcal{O}_{S 2}$. The resulting regions of $\bar{\kappa}_{U_{R}}-\kappa_{D}$ and $\bar{\kappa}_{U_{L}}-\kappa_{D}$ parameter space that accommodate the $\Delta A_{C P}$ measurement are shown for $m_{\phi}=10 \mathrm{GeV}$ in figure 7 . In this figure, we also show the constraints from $\operatorname{Br}(Z \rightarrow b \bar{b} \bar{b} b)$ and the single top quark production cross section from CDF [27], $\sigma^{\text {new }}$ (singletop) $<3.61 \mathrm{pb}$. We can see from the left panel of figure 7 that the entire $A_{C P}^{\exp }$ preferred parameter space for $\bar{\kappa}_{U_{R}}-\kappa_{D}$ has been excluded by the single top production cross section measurement, while the right panel shows that there is still allowed parameter space for $\bar{\kappa}_{U_{L}}-\kappa_{D}$.

Before continuing, we turn to the question of whether there are any further modes sensitive to the operators $\mathcal{O}_{V 2}$ and/or $\mathcal{O}_{S 2}$. Several criteria must be satisfied for any 
relevant observable. Due to the Yukawa suppression of $D$ decays from these operators, we consider only modes that are $C P$ violating to avoid competition with the dominant SM amplitude and involve $D \rightarrow K$ transitions to avoid additional Yukawa suppression. Furthermore, we have seen that $C P$ asymmetries generated in such transitions need to be measured to fairly high precision. There are only a small number of observables that are close to satisfying all of these criteria. The most promising ones are $A_{C P}\left(D^{0} \rightarrow K^{-} \pi^{+}\right)$ $\left(\mathcal{O}_{V 2}\right), A_{C P}\left(D^{0} \rightarrow K^{+} \pi^{-}\right)\left(\mathcal{O}_{S 2}\right)$, and $A_{C P}\left(D^{+} \rightarrow K^{+} K^{-} \pi^{+}\right)\left(\mathcal{O}_{V 2}, \mathcal{O}_{S 2}\right)$, where the operators in parentheses yield significant sensitivity. Modes involving $K^{0}$ are challenging to compute as they receive dominant contributions from $K-\bar{K}$ mixing, though they are among the most precisely measured. A full study of these additional observables is beyond the scope of this work.

\section{Discussion and conclusions}

In this paper, we have studied a class of models below the scale of electroweak symmetry breaking that lead to interesting $\Delta C=1$ and $\Delta T=1$ observables while remaining unconstrained by other flavor and precision observables. Despite these features, the models cannot be the full story: they require a UV completion to render them $\mathrm{SU}(2)_{L} \times \mathrm{U}(1)_{Y}$ invariant. In order to have $\mathcal{O}(1)$ couplings in the IR model, new states must be introduced at a scale no more than a factor of a few above the Higgs vacuum expectation value. We now briefly outline the general features of such UV completions.

There are only a limited number of possibilities in terms of the gauge charges of the particle that completes the theory. The completion must involve a Higgs field in order to break electroweak symmetry. If the particle is a boson, then it must have the same charges under SM gauge symmetries as a Higgs, ${ }^{3}$ as it must couple the relevant Higgs to the singlet $\phi$. If the particle is a fermion, then it must be a vector-like quark. It may be either an $\mathrm{SU}(2)_{L}$ doublet or singlet. Thus, for the up- and down-sectors, there are three possibilities for the field that implements the UV completion. In the up-sector, they are: $\Phi(1,2)_{1 / 2}$, $\Psi_{L, R}(3,2)_{1 / 6}, \Psi_{L, R}(3,1)_{2 / 3}$, while in the down-sector, they are: $\Phi(1,2)_{1 / 2}, \Psi_{L, R}(3,2)_{1 / 6}$, $\Psi_{L, R}(3,1)_{-1 / 3}$. Note that in the first two cases the same particle may be responsible for completing both the up- and the down-sectors. For each possible set of gauge charges, there are several possible flavor charges. The phenomenology of the model depends greatly on the specific flavor charge, as well as any other degrees of freedom that may appear near the scale of the UV completion. A complete study is beyond the scope of this work.

In summary, we have explored the phenomenology of the up-type quark sector in the MFV framework. Concentrating on the $C P$ violating effective operators, we have found interesting correlations between the $\Delta C=1$ and $\Delta T=1$ processes. If the flavor mediator has a mass below $\mathcal{O}(100 \mathrm{GeV})$, both processes can be probed at the LHC either in flavor physics related to $D$-meson decays or in the top quark physics related to top quark single and pair production. A further consequence of the existence of the light flavor mediator is a new decay channel for the top quark. This new decay channel, $t \rightarrow c+\phi$, would not

\footnotetext{
${ }^{3}$ We assume that there are only $\mathrm{SU}(2)_{L}$ doublet Higgses. Other representations are highly constrained by data.
} 
appear in existing flavor-changing-neutral-current decay searches such as $t \rightarrow c+Z$ because the top quark would appear as a "dijet" resonance for a $\phi$ mass below $\mathcal{O}(20 \mathrm{GeV})$. A fat jet analysis for the $\phi$ field from the top decay can therefore probe the up-quark sector.

\section{Acknowledgments}

We would like to thank Amarjit Soni and Jure Zupan for useful discussions and comments. Y. Bai is supported by startup funds from the UW-Madison. SLAC is operated by Stanford University for the US Department of Energy under contract DE-AC02-76SF00515.

\section{A Calculation of the partial width $\Gamma\left(Z \rightarrow q \bar{q}^{\prime} \phi\right)$}

The tree-level diagrams for this decay are shown in figure 4 . Note that there is a soft divergence in the limit $m_{\phi}=m_{q}=m_{q^{\prime}}=0$, so the calculation cannot be done in the limit where the final state masses all vanish. In this appendix, we present the differential width contributions for the decay, as well as the integrated width assuming that the quark masses vanish. Note that the assumption of $m_{q}=0$ is not necessarily sufficiently accurate for final states involving the $b$ quark due to the fact that $m_{\phi}$ is not much greater than $m_{b}$.

The amplitudes for the decay are given by

$$
\begin{aligned}
& \mathcal{M}_{1}=\bar{u}_{i}\left(i \kappa_{i j} P_{R}\right) \frac{i\left(\not p_{1}+\not p_{2}\right)+m_{j}}{\left(p_{1}+p_{2}\right)^{2}-m_{j}^{2}} \notin\left(i g_{L} P_{L}+i g_{R} P_{R}\right) v_{j}, \\
& \mathcal{M}_{2}=\bar{u}_{i} \notin\left(i g_{L} P_{L}+i g_{R} P_{R}\right) \frac{i\left(\not p_{2}+\not p_{3}\right)+m_{i}}{\left(p_{2}+p_{3}\right)^{2}-m_{i}^{2}}\left(i \kappa_{i j} P_{R}\right) v_{j},
\end{aligned}
$$

where $\kappa_{i j}$ is the coupling of quarks $i$ and $j$ to the scalar $\phi_{j i}$, including all factors of Yukawas, $g_{L, R}$ are the couplings of left-handed and right-handed quarks $q_{j}$ to $Z$, and $p_{1,2,3}$ are the momenta of $q_{i}, \phi_{j i}$, and $\overline{q_{j}}$ respectively.

The resulting differential width is given by

$$
\begin{aligned}
d \Gamma= & \frac{1}{(2 \pi)^{3}} \frac{1}{32 m_{Z}^{3}} \overline{|\mathcal{M}|^{2}} d m_{12}^{2} d m_{23}^{2}, \\
\overline{|\mathcal{M}|^{2}}= & \left(-g_{\mu \nu}+\frac{p_{\mu} p_{\nu}}{m_{Z}^{2}}\right)\left|\kappa_{i j}\right|^{2} \\
& \left\{\frac{\operatorname{Tr}\left[\left(\not p_{1}+m_{i}\right) P_{R}\left(\not \phi_{1}+m_{j}\right) \gamma^{\mu}\left(g_{L} P_{L}+g_{R} P_{R}\right)\left(\not p_{3}+m_{j}\right)\left(g_{L} P_{R}+g_{R} P_{L}\right) \gamma^{\nu}\left(\not q_{1}+m_{j}\right) P_{L}\right]}{\left(m_{12}^{2}-m_{j}^{2}\right)^{2}}\right. \\
& \frac{\operatorname{Tr}\left[\left(\not p_{1}+m_{i}\right) P_{R}\left(\not q_{1}+m_{j}\right) \gamma^{\mu}\left(g_{L} P_{L}+g_{R} P_{R}\right)\left(\not p_{3}+m_{j}\right) P_{L} \gamma^{\nu}\left(\not q_{2}+m_{i}\right) \gamma^{\nu}\left(g_{L} P_{L}+g_{R} P_{R}\right)\right]}{\left(m_{12}^{2}-m_{j}^{2}\right)\left(m_{23}^{2}-m_{i}^{2}\right)} \\
& \frac{\operatorname{Tr}\left[\left(\not p_{1}+m_{i}\right) P_{R}\left(\not \not_{2}+m_{i}\right) \gamma^{\mu}\left(g_{L} P_{L}+g_{R} P_{R}\right)\left(\not p_{3}+m_{j}\right)\left(g_{L} P_{R}+g_{R} P_{L}\right) \gamma^{\nu}\left(\not \phi_{1}+m_{j}\right) P_{L}\right]}{\left(m_{12}^{2}-m_{j}^{2}\right)\left(m_{23}^{2}-m_{i}^{2}\right)} \\
& \left.\frac{\operatorname{Tr}\left[\left(\not p_{1}+m_{i}\right) P_{R}\left(\not \phi_{2}+m_{i}\right) \gamma^{\mu}\left(g_{L} P_{L}+g_{R} P_{R}\right)\left(\not p_{3}+m_{j}\right) P_{L} \gamma^{\nu}\left(\not_{2}+m_{i}\right) \gamma^{\nu}\left(g_{L} P_{L}+g_{R} P_{R}\right)\right]}{\left(m_{23}^{2}-m_{i}^{2}\right)^{2}}\right\},
\end{aligned}
$$

where $q_{1}=p_{1}+p_{2}$ and $q_{2}=p_{2}+p_{3}$. 
The phase space integration yields an even more involved expression, but in the limit $m_{i}=m_{j}=0$, a relatively compact result emerges:

$$
\begin{aligned}
\Gamma\left(Z \rightarrow q_{i} \bar{q}_{j} \phi_{j i}\right)= & \frac{\alpha \kappa_{i j}^{2} m_{Z}}{576 \pi^{2} c_{w}^{2} s_{w}^{2}}\left\{\left(g_{L}^{2}+g_{R}^{2}\right)\left[-17+9 x+9 x^{2}-x^{3}-6 \log x-18 x \log x\right]\right. \\
& +g_{L} g_{R}\left[10+78 x-90 x^{2}+2 x^{3}+60 x \log x+36 x^{2} \log x+12 x^{2} \log ^{2} x\right. \\
& \left.\left.-48 x^{2} \log x \log (1+x)-24 x^{2}\left(2 \operatorname{Li}_{2}(-x)+\frac{\pi^{2}}{6}\right)\right]\right\}
\end{aligned}
$$

where $g_{L, R}=T_{3}-Q s_{w}^{2}$ and $x=m_{\phi}^{2} / m_{Z}^{2}$. This result provides a good approximation of the result in the massive quark case for physical quark masses and for $m_{\phi} \gtrsim 10 \mathrm{GeV}$.

\section{B Wilson coefficient running}

In this appendix, we present the details of the running of the Wilson coefficients for the various effective operators. In particular, we determine the one-loop anomalous dimension matrices for the coefficients of operators $\mathcal{O}_{V 2}$ and $\mathcal{O}_{S 2}$. The anomalous dimension matrices are defined such that

$$
\frac{d C_{i}}{d \mu}=\frac{\alpha_{s}}{4 \pi} \gamma^{T} C_{i},
$$

where $C_{i}$ 's are the Wilson coefficients of the set of operators that mix.

The operator $\mathcal{O}_{V 2}$ runs much like the operator $\mathcal{O}_{2}^{(1) p}$ in [56] except that it cannot receive contributions from penguin-like operators: those operators have a distinct chiral structure and mixing is forbidden by Lorentz symmetry. The only mixing is therefore with $\mathcal{O}_{V 1}$. The anomalous dimension matrix can be read off of the upper left $2 \times 2$ block of the anomalous dimension matrix for the operators $O_{i}^{(1)}$ in [56]:

$$
\gamma_{V}=\left(\begin{array}{cc}
-\frac{6}{N_{c}} & 6 \\
6 & -\frac{6}{N_{c}}
\end{array}\right)
$$

The operator $\mathcal{O}_{S 2}$ is identical in Lorentz and color structure to the operator $\mathcal{O}_{S 2}^{(1)}$ of [56] and therefore the anomalous dimension matrix is

$$
\gamma_{S}=\left(\begin{array}{cccc}
\frac{6-6 N_{c}^{2}}{N_{c}} & 0 & \frac{1}{N_{c}} & -1 \\
-6 & \frac{6}{N_{c}} & -\frac{1}{2} & \frac{2-N_{c}^{2}}{2 N_{c}} \\
\frac{48}{N_{c}} & -48 & \frac{2 N_{c}^{2}-2}{N_{c}} & 0 \\
-24 & \frac{48-24 N_{c}^{2}}{N_{c}} & 6 & \frac{4 N_{c}^{2}+2}{-N_{c}}
\end{array}\right) .
$$

\section{Hadronic matrix element estimation}

Recent work $[55,57]$ has demonstrated a consistent picture for observed $D$ meson physics within the SM under the assumption of a large deviation from naive factorization. On the other hand, this picture has yet to be confirmed by direct calculation of the hadronic matrix 
elements. It remains possible that there is enhanced $C P$ violation in the $D$ system due solely or partly to new physics contributions. The results regarding $D$ meson $C P$ violation in this paper therefore assume that naive factorization gives a reasonable estimate of the relative sizes of the various hadronic matrix elements contributing to $D$ meson decays. In this appendix, we present the details of the estimation used to calculate $\Delta A_{C P}$ in this paper, following the work of ref. [56].

Naive factorization is the assumption that a hadronic matrix element $\left\langle h^{+} h^{-}\left|\left(\bar{u} \Gamma_{1} q\right)\left(\bar{q} \Gamma_{2} c\right)\right| D^{0}\right\rangle$ can be reliably estimated by

$$
\left\langle h^{+} h^{-}\left|\left(\bar{u} \Gamma_{1} q\right)\left(\bar{q} \Gamma_{2} c\right)\right| D^{0}\right\rangle \approx\left\langle h^{+}\left|\left(\bar{u} \Gamma_{1} p\right)\right| 0\right\rangle\left\langle h^{-}\left|\left(\bar{p} \Gamma_{2} c\right)\right| D^{0}\right\rangle .
$$

Using (C.1), we can relate the hadronic matrix elements for the operators $\mathcal{O}_{V 2}$ and $\mathcal{O}_{S 2}$ to that for the leading SM operator $\mathcal{O}_{\mathrm{SM}} \equiv\left(\bar{u}_{L} \gamma^{\mu} q_{L}\right)\left(\bar{q}_{L} \gamma_{\mu} c_{L}\right)$. Under factorization, we can write:

$\left\langle h^{+} h^{-}\left|\left(\bar{u}_{L \alpha} \gamma^{\mu} q_{L \alpha}\right)\left(\bar{q}_{L \beta} \gamma_{\mu} c_{L \beta}\right)\right| D^{0}\right\rangle \approx-\frac{i}{2} \delta_{\alpha \alpha} \delta_{\beta \beta} p_{h^{+}} \cdot p_{h^{-}} f_{h} f_{+}^{D h^{-}}=-\frac{i}{2} N_{c}^{2} p_{h^{+}} \cdot p_{h^{-}} f_{h} f_{+}^{D h^{-}}$,

where we define $M_{1}^{\mu}=\left\langle h^{+}\left|\left(\bar{u}_{L \alpha} \gamma^{\mu} q_{L \alpha}\right)\right| 0\right\rangle$ (no sum over $\alpha$ ) and $M_{2}^{\mu}=\left\langle h^{-}\left|\left(\bar{q}_{L \alpha} \gamma^{\mu} c_{L \alpha}\right)\right| D^{0}\right\rangle$ (no sum over $\alpha$ ). Similarly, for the quark part of $\mathcal{O}_{V 2}$, we find

$\left\langle h^{+} h^{-}\left|\left(\bar{u}_{L \alpha} \gamma^{\mu} q_{L \beta}\right)\left(\bar{q}_{R \beta} \gamma_{\mu} c_{R \alpha}\right)\right| D^{0}\right\rangle \approx-\frac{i}{2} \delta_{\alpha \beta} \delta_{\alpha \beta} p_{h^{+}} \cdot p_{h^{-}} f_{h} f_{+}^{D h^{-}}=-\frac{i N_{c}}{2} p_{h^{+}} \cdot p_{h^{-}} f_{h} f_{+}^{D h^{-}}$.

Finally, we consider $\mathcal{O}_{S 2}$ :

$$
\begin{aligned}
\left\langle h^{+} h^{-}\left|\left(\bar{u}_{L \alpha} q_{R \beta}\right)\left(\bar{p}_{L \beta} c_{R \alpha}\right)\right| D^{0}\right\rangle & \approx \frac{i}{2} \delta_{\alpha \beta} \delta_{\alpha \beta} \frac{m_{h}^{2}}{m_{q}+m_{u}} f_{h} \frac{\left(p_{D}-p_{h^{-}}\right) \cdot p_{h^{-}}}{m_{c}-m_{s}} f_{+}^{D h^{-}} \\
& \approx i \frac{N_{c}}{2} \frac{m_{h}^{2}}{m_{c}\left(m_{q}+m_{u}\right)} p_{h^{+}} \cdot p_{h^{-}} f_{h} f_{+}^{D h^{-}} .
\end{aligned}
$$

Note that we relate the (pseudo-)scalar matrix elements to the (pseudo-)vector matrix elements using the Dirac equation. From these results, we obtain the following relations, assuming naive factorization:

$$
\mathcal{O}_{\mathrm{SM}}=N_{c} \mathcal{O}_{V 2}=\frac{2 N_{c}}{\chi_{f}} \mathcal{O}_{S 2}
$$

where $\chi_{K} \approx 2 m_{K}^{2} /\left[m_{c}\left(m_{s}+m_{u}\right)\right] \approx 4.2$ and $\chi_{\pi} \approx 2 m_{\pi}^{2} /\left[m_{c}\left(m_{d}+m_{u}\right)\right] \approx 2.8$.

Open Access. This article is distributed under the terms of the Creative Commons Attribution License which permits any use, distribution and reproduction in any medium, provided the original author(s) and source are credited.

\section{References}

[1] ATLAS collaboration, Observation of a new particle in the search for the Standard Model Higgs boson with the ATLAS detector at the LHC, Phys. Lett. B 716 (2012) 1 [arXiv:1207.7214] [INSPIRE]. 
[2] CMS collaboration, Observation of a new boson at a mass of $125 \mathrm{GeV}$ with the CMS experiment at the LHC, Phys. Lett. B 716 (2012) 30 [arXiv:1207.7235] [INSPIRE].

[3] R.S. Chivukula and H. Georgi, Composite Technicolor Standard Model, Phys. Lett. B 188 (1987) 99 [INSPIRE].

[4] G. D'Ambrosio, G. Giudice, G. Isidori and A. Strumia, Minimal flavor violation: an Effective field theory approach, Nucl. Phys. B 645 (2002) 155 [hep-ph/0207036] [INSPIRE].

[5] A.J. Buras and R. Fleischer, Bounds on the unitarity triangle, sin 2 beta and $K \rightarrow$ neutrino anti-neutrino decays in models with minimal flavor violation, Phys. Rev. D 64 (2001) 115010 [hep-ph/0104238] [INSPIRE].

[6] A.J. Buras, P.H. Chankowski, J. Rosiek and L. Slawianowska, Correlation between $\Delta M(s)$ and $B^{0}(s, d) \rightarrow \mu^{+} \mu^{-}$in supersymmetry at large tan beta, Phys. Lett. B 546 (2002) 96 [hep-ph/0207241] [INSPIRE].

[7] A.J. Buras, Relations between $\Delta M(s, d)$ and $B(s, d) \rightarrow \mu \bar{\mu}$ in models with minimal flavor violation, Phys. Lett. B 566 (2003) 115 [hep-ph/0303060] [INSPIRE].

[8] C. Bobeth et al., Upper bounds on rare $K$ and $B$ decays from minimal flavor violation, Nucl. Phys. B 726 (2005) 252 [hep-ph/0505110] [INSPIRE].

[9] M. Blanke, A.J. Buras, D. Guadagnoli and C. Tarantino, Minimal Flavour Violation Waiting for Precise Measurements of $\Delta M_{s}, S_{\psi \phi}, A_{S L}^{s},\left|V_{u b}\right|, \gamma$ and $B 0_{s, d} \rightarrow \mu^{+} \mu^{-}$, JHEP 10 (2006) 003 [hep-ph/0604057] [INSPIRE].

[10] M. Blanke and A.J. Buras, Lower bounds on $\Delta M(s, d)$ from constrained minimal flavour violation, JHEP 05 (2007) 061 [hep-ph/0610037] [INSPIRE].

[11] W. Altmannshofer, A.J. Buras and D. Guadagnoli, The MFV limit of the MSSM for low $\tan \beta$ : meson mixings revisited, JHEP 11 (2007) 065 [hep-ph/0703200] [INSPIRE].

[12] T. Hurth, G. Isidori, J.F. Kamenik and F. Mescia, Constraints on New Physics in MFV models: a Model-independent analysis of $\Delta F=1$ processes, Nucl. Phys. B 808 (2009) 326 [arXiv: 0807.5039] [INSPIRE].

[13] A.J. Buras, M.V. Carlucci, S. Gori and G. Isidori, Higgs-mediated FCNCs: natural Flavour Conservation vs. Minimal Flavour Violation, JHEP 10 (2010) 009 [arXiv: 1005.5310] [INSPIRE].

[14] B. Batell and M. Pospelov, Bs Mixing and Electric Dipole Moments in MFV, Phys. Rev. D 82 (2010) 054033 [arXiv: 1006.2127] [InSPIRE].

[15] T. Hurth and F. Mahmoudi, The Minimal Flavour Violation benchmark in view of the latest LHCb data, Nucl. Phys. B 865 (2012) 461 [arXiv:1207.0688] [INSPIRE].

[16] A.J. Buras and J. Girrbach, Stringent Tests of Constrained Minimal Flavour Violation through Delta F=2 Transitions, arXiv:1304.6835 [INSPIRE].

[17] E. Golowich, J. Hewett, S. Pakvasa and A.A. Petrov, Implications of $D^{0}-\bar{D}^{0}$ Mixing for New Physics, Phys. Rev. D 76 (2007) 095009 [arXiv: 0705.3650] [InSPIRE].

[18] V.D. Barger, J. Hewett and R. Phillips, New constraints on the charged Higgs sector in two Higgs doublet models, Phys. Rev. D 41 (1990) 3421 [INSPIRE].

[19] S. Faller, S. Gadatsch and T. Mannel, Minimal Flavour Violation and Anomalous Top Decays, arXiv:1304.2675 [INSPIRE]. 
[20] Y. Bai and A.E. Nelson, $C P$ Violating Contribution to $\Delta \Gamma$ in the $B_{s}$ System from Mixing with a Hidden Pseudoscalar, Phys. Rev. D 82 (2010) 114027 [arXiv: 1007.0596] [InSPIRE].

[21] J.L. Feng, T. Moroi, H. Murayama and E. Schnapka, Third generation familons, $b$ factories and neutrino cosmology, Phys. Rev. D 57 (1998) 5875 [inSPIRE].

[22] B. Pontecorvo, Mesonium and anti-mesonium, Sov. Phys. JETP 6 (1957) 429 [InSPIRE].

[23] Z. Maki, M. Nakagawa and S. Sakata, Remarks on the unified model of elementary particles, Prog. Theor. Phys. 28 (1962) 870 [InSPIRE].

[24] Z.-z. Xing, H. Zhang and S. Zhou, Updated Values of Running Quark and Lepton Masses, Phys. Rev. D 77 (2008) 113016 [arXiv:0712.1419] [INSPIRE].

[25] R.D. Kephart et al., A Measurement of the Dihadron Mass Continuum in Proton - Be Collisions and a Search for Narrow Resonances, Phys. Rev. Lett. 39 (1977) 1440 [inSPIRE].

[26] A. Martin, W. Stirling, R. Thorne and G. Watt, Parton distributions for the LHC, Eur. Phys. J. C 63 (2009) 189 [arXiv:0901.0002] [inSPIRE].

[27] CDF collaboration, Measurement of Single Top Quark Production in $7.5 \mathrm{fb}^{-1}$ of CDF Data Using Neural Networks, CDF/PUB/TOP/PUBLIC/10793 (2013).

[28] CMS collaboration, Measurement of the single-top t-channel cross section in pp collisions at centre-of-mass energy of $8 \mathrm{TeV}$, CMS-PAS-TOP-12-011

[29] ATLAS collaboration, Measurement of the top quark pair production cross section in the single-lepton channel with ATLAS in proton-proton collisions at $8 \mathrm{TeV}$ using kinematic fits with b-tagging, ATLAS-CONF-2012-149 (2012).

[30] CDF and D0 collaboration, Measurement of the top quark pair production cross section at Tevatron, http://www-cdf.fnal.gov/physics/new/top/2009/singletop/TeVcombination/.

[31] A. Czarnecki and K. Melnikov, Two loop QCD corrections to top quark width, Nucl. Phys. B 544 (1999) 520 [hep-ph/9806244] [InSPIRE].

[32] K. Chetyrkin, R. Harlander, T. Seidensticker and M. Steinhauser, Second order QCD corrections to $\Gamma(t \rightarrow W b)$, Phys. Rev. D 60 (1999) 114015 [hep-ph/9906273] [INSPIRE].

[33] J. Gao, C.S. Li and H.X. Zhu, Top Quark Decay at Next-to-Next-to Leading Order in QCD, Phys. Rev. Lett. 110 (2013) 042001 [arXiv: 1210.2808] [INSPIRE].

[34] M. Brucherseifer, F. Caola and K. Melnikov, $\mathcal{O}\left(\alpha_{s}^{2}\right)$ corrections to fully-differential top quark decays, JHEP 04 (2013) 059 [arXiv:1301.7133] [INSPIRE].

[35] D0 collaboration, V.M. Abazov et al., Determination of the width of the top quark, Phys. Rev. Lett. 106 (2011) 022001 [arXiv:1009.5686] [InSPIRE].

[36] ECFA/DESY LC Physics Working Group collaboration, J. Aguilar-Saavedra et al., TESLA: the Superconducting electron positron linear collider with an integrated $x$-ray laser laboratory. Technical design report. Part 3. Physics at an $e^{+} e^{-}$linear collider, hep-ph/0106315 [INSPIRE].

[37] LHC/LC Study Group collaboration, G. Weiglein et al., Physics interplay of the LHC and the ILC, Phys. Rept. 426 (2006) 47 [hep-ph/0410364] [INSPIRE].

[38] ILD Concept Group - Linear Collider collaboration, T. Abe et al., The International Large Detector: letter of Intent, arXiv:1006.3396 [INSPIRE].

[39] H. Aihara et al., SiD Letter of Intent, arXiv:0911.0006 [INSPIRE]. 
[40] Particle Data Group collaboration, J. Beringer et al., Review of Particle Physics (RPP), Phys. Rev. D 86 (2012) 010001 [inSPIRE].

[41] DELPHI collaboration, P. Abreu et al., Measurement of the rate of $b \bar{b} b \bar{b}$ events in hadronic $Z$ decays and the extraction of the gluon splitting into bb, Phys. Lett. B 462 (1999) 425 [INSPIRE].

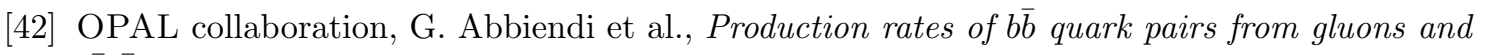
$b \bar{b} b \bar{b}$ events in hadronic Z0 decays, Eur. Phys. J. C 18 (2001) 447 [hep-ex/0010029] [INSPIRE].

[43] G. Isidori, J.F. Kamenik, Z. Ligeti and G. Perez, Implications of the LHCb Evidence for Charm CP-violation, Phys. Lett. B 711 (2012) 46 [arXiv:1111.4987] [INSPIRE].

[44] Y. Grossman, A.L. Kagan and Y. Nir, New physics and CP-violation in singly Cabibbo suppressed D decays, Phys. Rev. D 75 (2007) 036008 [hep-ph/0609178] [INSPIRE].

[45] BABAR collaboration, B. Aubert et al., Search for CP-violation in the decays $D^{0} \rightarrow K^{-} K^{+}$ and $D^{0} \rightarrow \pi^{-} \pi^{+}$, Phys. Rev. Lett. 100 (2008) 061803 [arXiv:0709.2715] [InSPIRE].

[46] Belle collaboration, B. Ko, Direct CP-violation in charm at Belle, arXiv:1212.1975 [INSPIRE].

[47] CDF collaboration, T. Aaltonen et al., Measurement of the difference of CP-violating asymmetries in $D^{0} \rightarrow K^{+} K^{-}$and $D^{0} \rightarrow \pi^{+} \pi^{-}$decays at $C D F$, Phys. Rev. Lett. 109 (2012) 111801 [arXiv:1207.2158] [INSPIRE].

[48] LHCB collaboration,, A search for time-integrated CP-violation in $D^{0} \rightarrow K^{-} K^{+}$and $D^{0} \rightarrow \pi^{-} \pi^{+}$decays, CERN-LHCb-CONF-2013-003.

[49] LHCB collaboration, Search for direct CP violation in $D^{0} \rightarrow h^{-} h^{+}$modes using semileptonic $B$ decays, Phys. Lett. B 723 (2013) 33 [arXiv:1303.2614] [INSPIRE].

[50] M. Gersabeck, Combination of Direct and Indirect CP Violation, http://www.slac.stanford.edu/xorg/hfag/charm/March13/DCPV/direct_indirect_cpv.html (2013).

[51] T. Blum et al., Lattice determination of the $K \rightarrow(\pi \pi)_{I=2}$ Decay Amplitude $A_{2}$, Phys. Rev. D 86 (2012) 074513 [arXiv:1206.5142] [INSPIRE].

[52] RBC, UKQCD collaborations, P. Boyle et al., Emerging understanding of the $\Delta I=1 / 2$ Rule from Lattice $Q C D$, arXiv:1212.1474 [INSPIRE].

[53] M. Golden and B. Grinstein, Enhanced CP-violations in Hadronic Charm Decays, Phys. Lett. B 222 (1989) 501 [inSPIRE].

[54] D. Atwood and A. Soni, Searching for the Origin of CP-violation in Cabibbo Suppressed D-meson Decays, arXiv:1211.1026 [INSPIRE].

[55] Y. Grossman and D.J. Robinson, SU(3) Sum Rules for Charm Decay, JHEP 04 (2013) 067 [arXiv: 1211.3361] [INSPIRE].

[56] W. Altmannshofer, R. Primulando, C.-T. Yu and F. Yu, New Physics Models of Direct CP-violation in Charm Decays, JHEP 04 (2012) 049 [arXiv: 1202.2866] [InSPIRE].

[57] J. Brod, Y. Grossman, A.L. Kagan and J. Zupan, A Consistent Picture for Large Penguins in $D \rightarrow \pi^{+} \pi^{-}, K^{+} K^{-}$, JHEP 10 (2012) 161 [arXiv:1203.6659] [INSPIRE]. 